\title{
Ex vivo hydrodynamics after central and paracommissural edge-to-edge technique: A further step toward transcatheter tricuspid repair?
}

\author{
Sina Stock, MD, ${ }^{\mathrm{a}}$ Heidemarie Bohm, MD, ${ }^{\mathrm{a}}$ Michael Scharfschwerdt, PhD, ${ }^{\mathrm{a}}$ Doreen Richardt, MD, ${ }^{\mathrm{a}}$ \\ Roza Meyer-Saraei, PhD, ${ }^{b}$ Stanislav Tsvelodub, MD, ${ }^{a}$ and Hans-Hinrich Sievers, MD $^{\mathrm{a}}$
}

\section{ABSTRACT}

Objectives: Transcatheter approaches in heart valve disease became tremendously important and are currently established in the aortic position, but transcatheter tricuspid repair is still in its beginning and remains challenging. Replicating the surgical edge-to-edge technique, for example, with the MitraClip System (Abbott Vascular, Santa Clara, Calif), represents a promising option and has been reported successfully in small numbers of cases. However, up to now, few data considering the edge-to-edge technique as a transcatheter approach are available. This study aims to determine the ex vivo hydrodynamics after the central and paracommissural edge-to-edge technique in different pathologies.

Methods: Because of basal or apical dislocation of papillary muscles, leaflet prolapse or tethering was simulated in porcine tricuspid valves mounted on a flexible holding device. Central and paracommissural edge-to-edge techniques were evaluated successively in these pathologies. Regurgitant volume and mean transvalvular gradient were determined in a pulse duplicator.

Results: In this ex vivo model, the isolated edge-to-edge technique reduced tricuspid regurgitation. In the prolapse model, regurgitant volume decreased significantly after central edge-to-edge technique (from $49.4 \pm 13.6 \mathrm{~mL} / \mathrm{stroke}$ to $39.3 \pm 14.1 \mathrm{~mL} /$ stroke). In the tethering model, both the central and the paracommissural edge-to-edge techniques led to a significant decrease (from $48.7 \pm 13.9$ to $43.6 \pm 15.6$ and to $41.1 \pm 13.8 \mathrm{~mL} /$ stroke). In all cases, the reduction of regurgitant volume was achieved at the cost of significantly increased mean transvalvular gradient.

Conclusions: This study provides a reduction of tricuspid regurgitation after the edge-to-edge technique in the specific experimental setup. Whether this reduction is sufficient to treat tricuspid regurgitation successfully in clinical practice remains to be established. Transcatheter approaches need to be evaluated further, probably with regard to concomitant annuloplasty for higher reduction of tricuspid regurgitation. (J Thorac Cardiovasc Surg 2018;155:949-55)

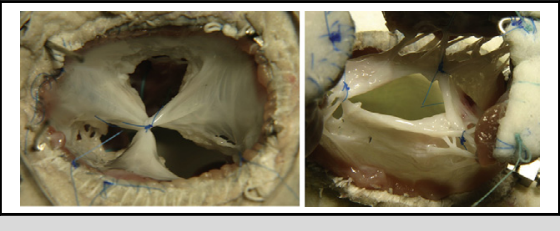

Photograph of different EETs in tricuspid valve preparations.

\section{Central Message}

This ex vivo model showed significant reduction but not elimination of TR, implicating potential consequences for transcatheter techniques.

\section{Perspective}

Transcatheter tricuspid repair is still in its beginning. A clinically applied device is the MitraClip System (Abbott Vascular, Santa Clara, Calif) replicating the surgical EET, but few data evaluating this strategy are available. This ex vivo study investigated different localizations of edge-to-edge repair in different pathologies, aiming to provide further data with potential implications for transcatheter techniques.

See Editorial Commentary page 956.

\footnotetext{
From the a Department of Cardiac and Thoracic Vascular Surgery Luebeck, and ${ }^{\mathrm{b}}$ Department of Cardiology, Angiology and Intensive Care Medicine Luebeck, German Centre for Cardiovascular Research (DZHK), Partner site Hamburg/Kiel/Luebeck, University Medical Centre of Schleswig-Holstein, Luebeck, Germany.

Received for publication May 9, 2017; revisions received Sept 29, 2017; accepted for publication Oct 20, 2017; available ahead of print Dec 6, 2017.

Address for reprints: Hans-Hinrich Sievers, MD, Department of Cardiac and Thoracic Vascular Surgery Luebeck, University Medical Centre of Schleswig-Holstein, Ratzeburger Allee 160, 23538 Luebeck, Germany (E-mail: Hans-Hinrich.Sievers@ uksh.de).

$0022-5223 / \$ 36.00$

Copyright (c) 2017 by The American Association for Thoracic Surgery

https://doi.org/10.1016/j.jtcvs.2017.10.068
}

In recent years, functional tricuspid regurgitation (FTR) as a cause of a patient's morbidity and mortality has become an issue of particular interest. Although severe tricuspid

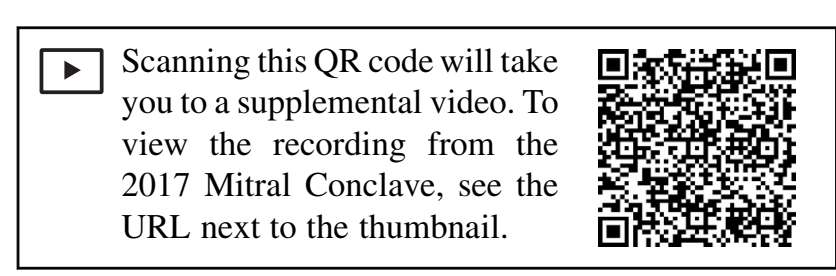




$$
\begin{aligned}
& \text { Abbreviations and Acronyms } \\
& \qquad \mathrm{EET}=\text { edge-to-edge technique } \\
& \mathrm{FTR}=\text { functional tricuspid regurgitation } \\
& \delta \mathrm{p} \quad=\text { mean transvalvular gradient } \\
& \mathrm{PAH}=\text { pulmonary artery hypertension } \\
& \mathrm{TR}=\text { tricuspid regurgitation } \\
& \mathrm{v}_{\mathrm{r}} \quad=\text { regurgitant volume }
\end{aligned}
$$

regurgitation (TR) can result in serious clinical symptoms, it often remains undertreated.

FTR is predominantly caused secondary to left heart disease, especially mitral regurgitation, mitral stenosis, and aortic stenosis, as well as to concomitant pulmonary artery hypertension (PAH). In addition, chronic atrial fibrillation favors tricuspid annular dilation and therefore the occurrence of FTR. ${ }^{1,2}$ Increased right ventricular pressure and volume overload due to left heart disease and PAH induce tricuspid annular dilation and enlargement of the right ventricle, leading to leaflet tethering caused by dislocation of the papillary muscles and further worsening FTR. Because annular dilation and ventricular enlargement are of progressive character, FTR will increase over time if it remains untreated. ${ }^{2-5}$ The need for surgical interventions of FTR, which represents approximately $90 \%$ of all severe TR pathologies, ${ }^{1,6}$ has been underestimated for a long time because of the concept that TR would recede once the primary left heart disease had been treated. ${ }^{4,5}$ Several reports of different research groups over the past few years refuted this hypothesis and revealed the deteriorating character of TR, leading to an extension of the indication for surgical tricuspid repair. ${ }^{1-5}$ According to the most recent guidelines, surgical treatment of TR during left heart surgery is also recommended in patients with tricuspid annulus dilation and nonsevere $\mathrm{TR}^{7,8}$

Nevertheless, the indications for tricuspid valve surgery in individual cases remain a challenge in clinical practice, and the recurrence of moderate to severe TR can be as high as $60 \%$ at 5 years. ${ }^{9}$ Because reoperation for tricuspid repair in these patients is associated with a mortality as high as $35 \%,{ }^{1,3,10,11}$ there is a need for less-invasive treatment alternatives, such as transcatheter approaches.

Today, transcatheter techniques are an established alternative to surgery in aortic valve disease and emerging in mitral valve disease, but still in their beginning in tricuspid valve disease. Several devices are in clinical application to treat severe TR and show promising results, but because of small case numbers, these results are preliminary and there is still no structured evaluation of the different techniques. An appealing concept seems to be the imitation of the surgical edge-to-edge technique (EET), because it shows satisfactory results in surgical midterm follow-up. ${ }^{12}$ The transcatheter approach in clinical practice to mimic this technique is performed with the MitraClip System (Abbott Vascular, Santa Clara, Calif), reported by different research groups to reduce severe TR significantly. ${ }^{13-19}$ However, this technique has been used only in small numbers of patients and still needs further evaluation before broad clinical application. In particular, only few studies investigated postinterventional transvalvular gradients (mean transvalvular gradient $[\delta \mathrm{p}]),{ }^{3,14,15}$ which might be elevated because of a reduced effective orifice area.

This study aims to investigate EET in a controlled ex vivo setting, evaluating regurgitant volume $\left(\mathrm{v}_{\mathrm{r}}\right)$ and $\delta \mathrm{p}$, considering 2 different techniques (central and paracommissural EET), as well as 2 different pathologies (leaflet prolapse and tethering) and simulating PAH, to evaluate the clinical applicability of EET with regard to transcatheter tricuspid repair.

\section{MATERIALS AND METHODS Tricuspid Valves}

Porcine tricuspid valve complexes $(n=12)$ were dissected out of porcine hearts, including the tricuspid annulus, leaflets, chordae tendineae, and papillary muscles. All porcine hearts were obtained from animals of the butchery Pirdzuhn (Todesfelde, Germany). The tricuspid valve preparations were mounted on a supporting device, consisting of an anatomically shaped holding ring for the fixation of the tricuspid valve annulus, heightadjustable frames for the attachment of the papillary muscles to simulate the different pathologies, and a spacer to stabilize the bottoms of the frames during the measurements (Figure 1).

\section{Simulation of Different Pathologies}

Underlying pathologies causing TR in this ex vivo study were leaflet prolapse and tethering. Both pathologies were imitated by dislocating the papillary muscles through shifting the height-adjustable frames, in case of prolapse in the basal direction and of tethering in the apical direction.

To validate the design of this ex vivo model, $\mathrm{v}_{\mathrm{r}}$ of the native and pathologically modified tricuspid valves was determined in a pulse duplicator. This validation aimed to review whether the simulations of both pathologies (prolapse and tethering) result in significantly higher $\mathrm{v}_{\mathrm{r}}$ compared with the native valve preparations and if a $v_{\mathrm{r}}$ similar to clinical conditions found in severe TR can be achieved. In this ex vivo study, the recommendations of the European Association of Echocardiography for the assessment of TR served as the basis for the definition of clinical conditions. According to these recommendations, TR is graded severe if $v_{r}$ exceeds $45 \mathrm{~mL} /$ stroke. $^{20}$

\section{Pulse Duplicator}

The physiologic circulation was imitated by a pulse duplicator allowing for the evaluation of hydrodynamic parameters. ${ }^{21}$ It enables the adjustment of different cardiac output volumes, afterload, and heart rates. A highspeed camera in front of the chamber containing the tricuspid valve preparations made a visual observation and pictures possible.

\section{Experimental Procedure}

In each tricuspid valve preparation, measurements were performed on the native tricuspid valve, both pathologies (prolapse and tethering), and central as well as paracommissural EET in both pathologies successively (Figure 2), taking the mean value.

A tricuspid valve preparation, mounted on the supporting device, was inserted into the pulse duplicator and hydrodynamic parameters $\left(\mathrm{v}_{\mathrm{r}}\right.$ and 

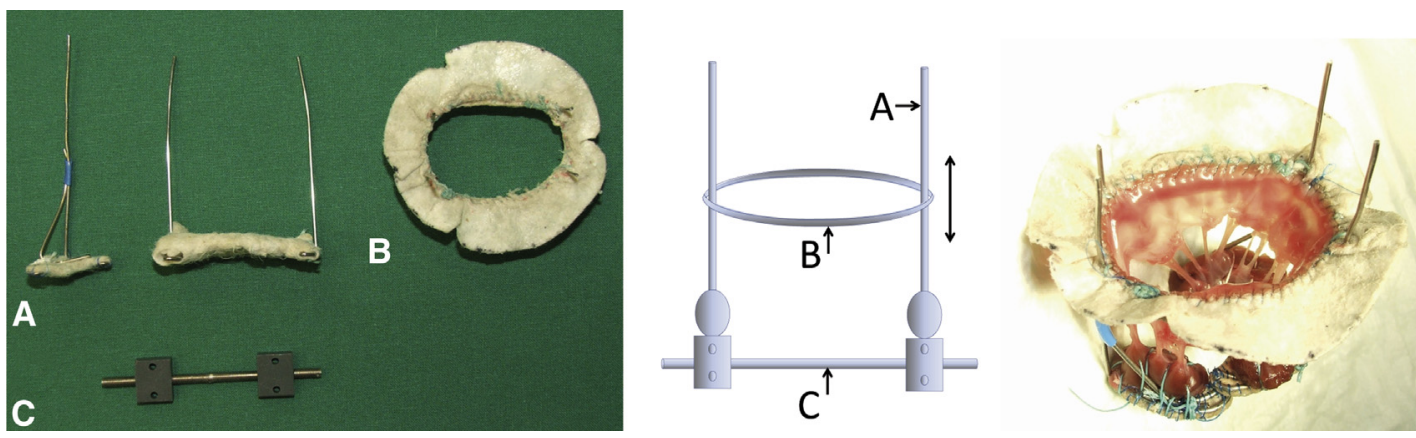

FIGURE 1. Tricuspid valve preparation and holding device. Left and middle: Photograph and schematic drawing of the holding device. It consists of height-adjustable frames for the attachment of the papillary muscles (A), which are connected to an anatomically shaped holding ring (B) serving for annular fixation of the tricuspid valve preparations and a spacer (C) that is attached to the bottoms of the papillary muscle frames to stabilize them during the measurements. Right: Tricuspid valve complex mounted on the holding device.

$\delta$ p) were determined. Afterward, the native tricuspid valve complex was explanted, leaflet prolapse was induced by height adjustment of the papillary muscle frames to the basal direction, that is, shifting the frames toward the annular ring, and the measurements were repeated. Afterward, central and paracommissural EET were performed successively and hydrodynamic parameters were determined (Video 1). Central EET was done by connecting the respective mid points of each leaflet's free edge through a suture, whereas paracommissural EET was performed by suturing the commissural edges of adjacent leaflets near the chordae interconnections (Figure 3).

For the next experimental run, leaflet tethering was induced by height adjustment of the papillary muscle frames to apical direction, that is, shifting the frames off the annular ring, on the same tricuspid valve preparation and the measurements were repeated according to the protocol.

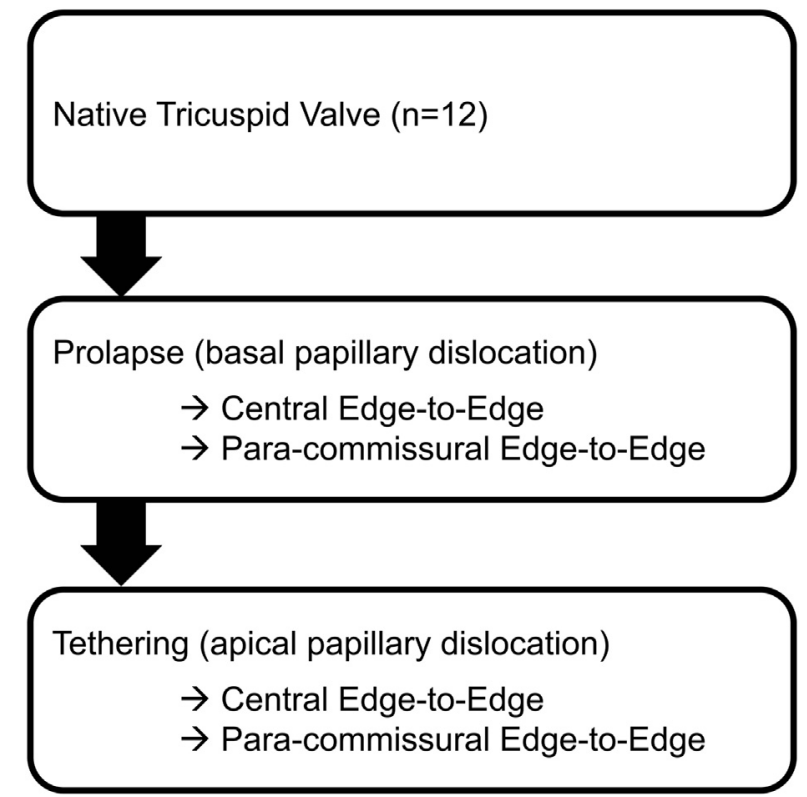

FIGURE 2. Trial protocol. Flow chart of the trial protocol. Prolapse and tethering were simulated because of papillary muscle displacement in every native tricuspid valve successively. Afterward, the central and paracommissural EET was performed successively. Measurements were performed at every single step.
During the measurements, systolic pressure was $60 \mathrm{~mm} \mathrm{Hg}$ and diastolic pressure was $20 \mathrm{~mm} \mathrm{Hg}$, simulating PAH. Stroke rate was 64 beats/min and stroke volume $70 \mathrm{~mL}$. The test solution was represented by a physiologic saline $(0.9 \%)$ with a density of $1.0046 \mathrm{~g} / \mathrm{cm}^{3}$ and a dynamic viscosity of $0.9 \mathrm{mPa} \cdot \mathrm{s}$ at an ambient temperature of $20^{\circ} \mathrm{C}$. Measurements were performed over a period of 10 heart cycles according to the standard of testing cardiac valve prostheses (ISO 5840:2005).

\section{Technique of Measurement}

The volume flow over the tricuspid valves was recorded by a sensor of an ultrasonic flowmeter TS-410 (Transonic System Inc, Ithaca, NY) mounted on the inflow line to the chamber containing the tricuspid valves. The sensor works bidirectionally with a resolution of $2 \mathrm{~mL} / \mathrm{min}$ and records flow rates up to $20 \mathrm{~L} / \mathrm{min}$.

Two capacitive pressure transducers Envec Ceracore M (Endress + Hauser, Maulburg, Germany), calibrated to a measuring range of -20 to $+160 \mathrm{~mm} \mathrm{Hg}$ and a resolution of $0.02 \mathrm{~mm} \mathrm{Hg}$, recorded the pressure before and after the tricuspid valves, allowing for the evaluation of $\delta \mathrm{p}$.

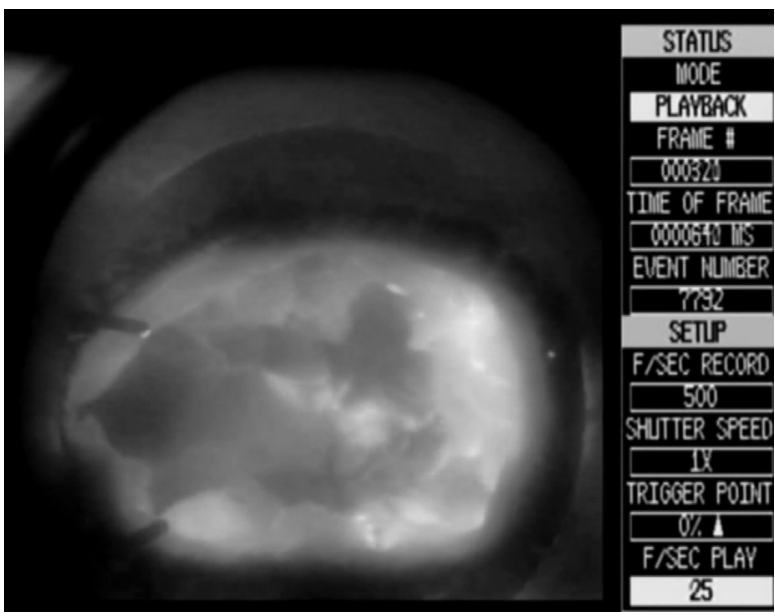

VIDEO 1. Exemplary depiction of the experimental procedure. Recordings of measurements in the pulse duplicator by the integrated highspeed camera show the atrial view on a native tricuspid valve preparation, a tricuspid valve preparation with prolapse simulation, and central and paracommissural edge-to-edge repair on the prolapsing valve. Video available at: http://www.jtcvsonline.org/article/S0022-5223(17)32397-8/fulltext. 

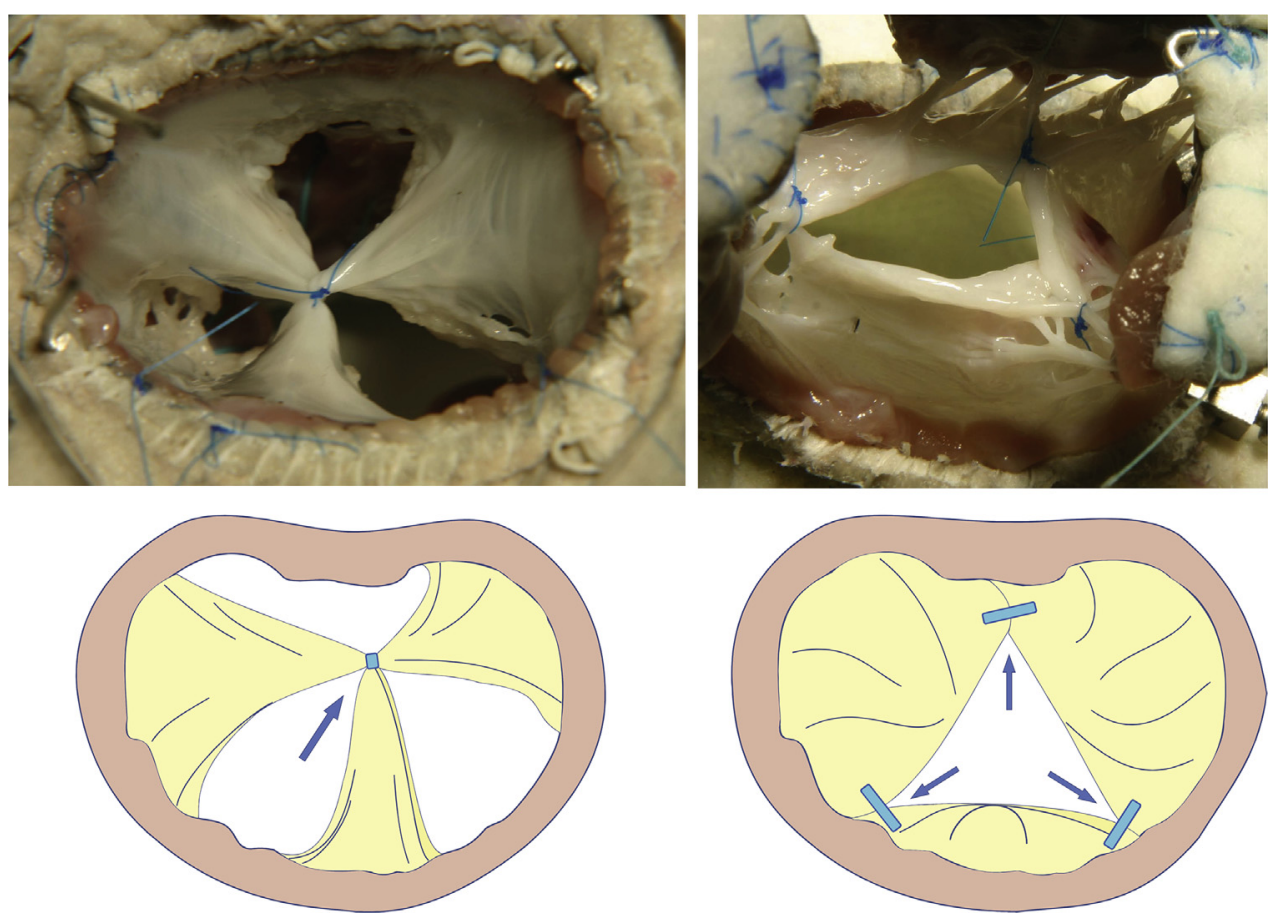

FIGURE 3. Central and paracommissural EET. Left: Atrial view on the central EET in a porcine tricuspid valve preparation mounted on the holding device and schematic drawing. The respective midpoints of each leaflet's free edge are connected by a suture. Right: Ventricular view on the paracommissural EET in a porcine tricuspid valve preparation mounted on the holding device and schematic drawing. The commissural edges of adjacent leaflets are sutured near the chordae interconnections.

A high-speed camera Motionscope HR-1000 (Redlake Imaging Corp, Morgan Hill, Calif) in front of the chamber containing the tricuspid valves recorded the characteristics of motion with 500 pictures per second. Video recordings and flow, as well as pressure measurements, were started simultaneously by using trigger signals.

\section{Analysis and Statistics}

All data were presented as mean value \pm standard deviation and median with the corresponding range in parentheses. Pre- and postinterventional data were compared using the Wilcoxon signed-rank test with the level of significance set at .05. Results were analyzed with the SPSS statistics software version 22.0.0.2 (IBM Corp, Armonk, NY).

\section{RESULTS}

\section{Validation of the Model}

The statistical evaluation indicated a significantly higher $\mathrm{v}_{\mathrm{r}}$ in both pathologies (prolapse: $49.4 \pm 13.6 \mathrm{~mL} /$ stroke and $51.4 \mathrm{~mL} /$ stroke [23.7-72.3 mL/stroke], respectively, tethering: $48.7 \pm 13.9 \mathrm{~mL} /$ stroke and $48.4 \mathrm{~mL} /$ stroke [16.7-69.3], respectively, both $P \leq .005)$ compared with the native valves $(27.3 \pm 8.5 \mathrm{~mL} / \mathrm{stroke}$ and $25 \mathrm{~mL} /$ stroke [15.4-39.7 mL/stroke], respectively). Furthermore, because $v_{r}$ in both pathologies exceeded the cutoff value of $45 \mathrm{~mL} /$ stroke defining severe TR according to the European Association of Echocardiography, ${ }^{20}$ the ex vivo model met the criteria of clinical conditions. In summary, the experimental design was proven valid for the conduction of this study.

\section{Regurgitant Volume}

Paracommissural EET resulted in a significant decrease of $\mathrm{v}_{\mathrm{r}}$ in the tethering group only $(41.1 \pm 13.8 \mathrm{~mL} / \mathrm{stroke}$ and $43.3 \mathrm{~mL} / \mathrm{stroke}$ [14.8-58.3 mL/stroke], respectively, $P=.028)$. After central EET, $\mathrm{v}_{\mathrm{r}}$ decreased significantly in the prolapse as well as the tethering group (prolapse: $39.3 \pm 14.1 \mathrm{~mL} / \mathrm{stroke}$ and $38.2 \mathrm{~mL} / \mathrm{stroke}$ [14.6$62.8 \mathrm{~mL} / \mathrm{stroke}]$, respectively, $P=.007$; tethering:

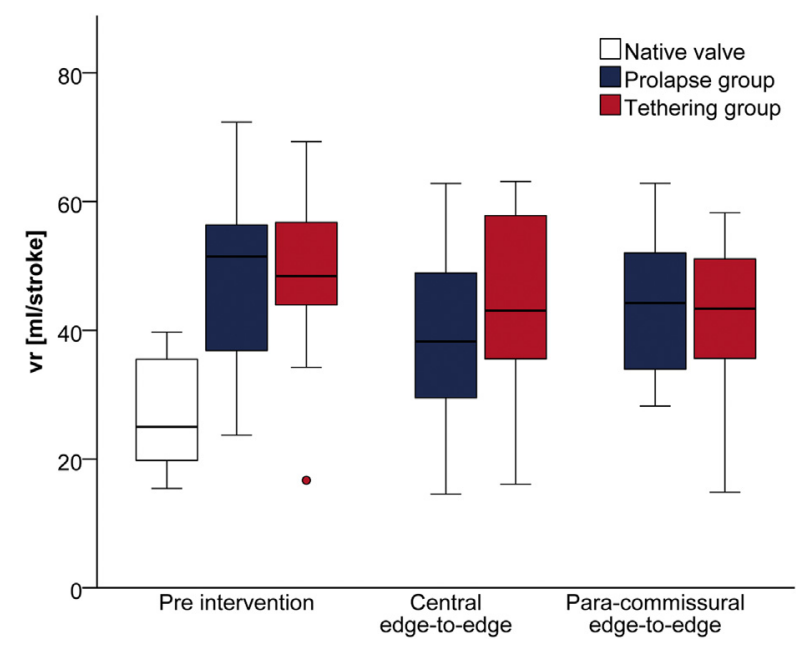

FIGURE 4. $\mathrm{v}_{\mathrm{r}} \cdot{ }^{\circ}$ Statistical outliers, defined as values that do not fall in the inner fences. $V_{r}$, Regurgitant volume. 


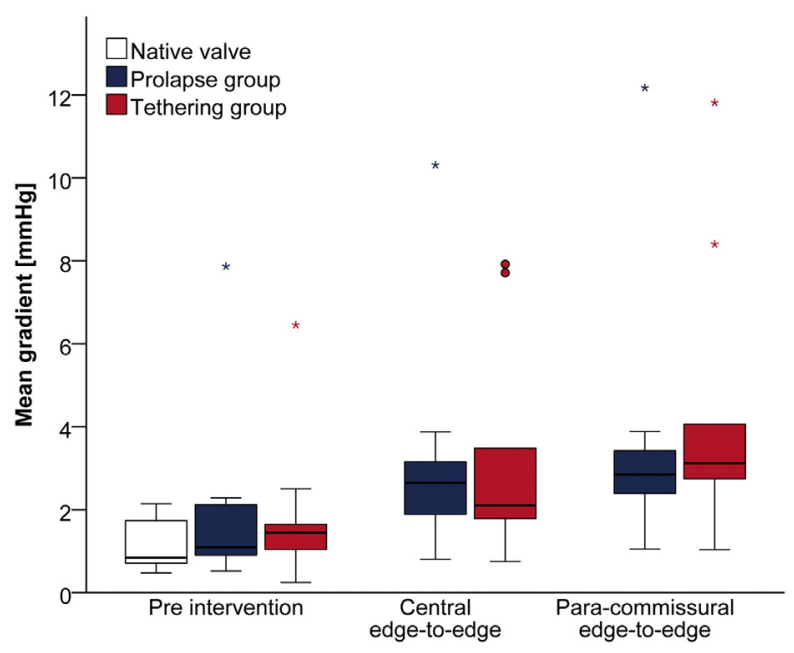

FIGURE 5. $\delta$ p. *Values more than 3 times the height of the boxes. ${ }^{\circ}$ Statistical outliers, defined as values that do not fall in the inner fences.

$43.6 \pm 15.6 \mathrm{~mL} /$ stroke and $43.1 \mathrm{~mL} /$ stroke [16.1-63.1 mL/ stroke], respectively, $P=.041)$. The most promising result with the highest decrease of $\mathrm{v}_{\mathrm{r}}$ was seen after central EET in the prolapse group (Figure 4).

\section{Mean Transvalvular Gradient}

Before intervention, $\delta$ p was $1.9 \pm 2.1 \mathrm{~mm} \mathrm{Hg}$ and $1.1 \mathrm{~mm}$ $\mathrm{Hg}(0.5-7.9 \mathrm{~mm} \mathrm{Hg})$, respectively, in the prolapse group and $1.9 \pm 1.7 \mathrm{~mm} \mathrm{Hg}$ and $1.4 \mathrm{~mm} \mathrm{Hg}(0.2-6.5 \mathrm{~mm} \mathrm{Hg})$, respectively, in the tethering group, without significant difference to the native tricuspid valves. Central and paracommissural EET led to significant increase of $\delta \mathrm{p}$ in both pathologies (prolapse: $3.1 \pm 2.5 \mathrm{~mm} \mathrm{Hg}$ and $2.6 \mathrm{~mm} \mathrm{Hg}$ [0.8-10.3 mm $\mathrm{Hg}$ ], respectively, $P=.005$ for central and $3.7 \pm 3.1 \mathrm{~mm}$ $\mathrm{Hg}$ and $2.8 \mathrm{~mm} \mathrm{Hg}$ [1.1-12.2 $\mathrm{mm} \mathrm{Hg}$, respectively, $P=.008$ for paracommissural EET; tethering: $3.3 \pm 2.5 \mathrm{~mm} \mathrm{Hg}$ and $2.1 \mathrm{~mm} \mathrm{Hg}$ [0.8-7.9 $\mathrm{mm} \mathrm{Hg}]$, respectively, $P=.008$ for central and $4.4 \pm 3.3 \mathrm{~mm} \mathrm{Hg}$ and $3.1 \mathrm{~mm}$ $\mathrm{Hg}$ [1-11.8 $\mathrm{mm} \mathrm{Hg}$ ], respectively, $P=.017$ for paracommissural EET). The highest increase in $\delta$ p was determined after paracommissural EET in the tethering group (Figure 5).

\section{DISCUSSION}

This study aimed to investigate further the use of central and paracommissural EET under the condition of PAH in 2 different pathologies of severe TR (prolapse and tethering) in a controlled ex vivo setting. Both EETs led to a significant decrease of $\mathrm{v}_{\mathrm{r}}$, but at the cost of a significant increase in $\delta \mathrm{p}$. The most effective technique was central EET in the prolapse group, providing the highest decrease of $\mathrm{v}_{\mathrm{r}}$ and lowest increase of $\delta \mathrm{p}$.

Transcatheter techniques for the treatment of severe TR are of particular interest and strongly needed to reduce mortality and improve patients' clinical outcome. Several transcatheter devices, some specifically developed for the use in tricuspid valve pathologies and some originally developed for other valve pathologies, are currently under development or at early stages in clinical application and need further evaluation. ${ }^{3,4,22}$ The clinically applied transcatheter device for the imitation of the surgical EET in the tricuspid valve is the MitraClip System, which was initially developed and is used to treat mitral regurgitation. Because midterm follow-up data of the surgical EET in tricuspid repair revealed satisfactory results ${ }^{12}$ and interventional cardiologists are familiar with the MitraClip System, its use to treat severe TR in patients with high surgical risk is an appealing concept. However, only few data evaluating this strategy are available.

This ex vivo study, conducted on a porcine model, investigates the feasibility of this transcatheter approach, simulating severe TR using papillary muscle displacement.

\section{Reduction of the Regurgitant Volume}

The application of EET led to a significant reduction of $\mathrm{v}_{\mathrm{r}}$ in 3 of the 4 tested groups; only paracommissural EET in the prolapse group was unsuccessful. Up to now, central EET grasping all 3 tricuspid leaflets, also known as the "clover technique," has been evaluated only in surgical approaches, but not with regard to transcatheter techniques. Few clinical studies reported a significant reduction of $\mathrm{v}_{\mathrm{r}}$ in patients with severe TR to trace or mild TR, ${ }^{12,23-25}$ but it has to be highlighted that the clover technique was performed as a concomitant technique to annuloplasty, apart from rare exceptions. Among the different author groups, Lapenna and colleagues ${ }^{12}$ evaluated the largest patient cohort, characterized by severe TR due to complex lesions including leaflet prolapse or tethering, comparable to the tricuspid valve pathologies simulated in this study. The authors report a satisfactory midterm outcome with significant reduction from severe TR to trace TR after central EET. These findings can be confirmed only partially by the results of this ex vivo study. Although central EET led to a significant reduction in $\mathrm{v}_{\mathrm{r}}$ in the prolapse and tethering groups, the extent of this reduction appears too small to replicate the clinical results of Lapenna and colleagues. ${ }^{12}$ An explanation for this discrepancy might be the absence of concomitant annuloplasty. Also, the PAH simulated in this ex vivo model might lead to increased $\mathrm{v}_{\mathrm{r}}$ compared with other studies.

Considering the feasibility of central EET using transcatheter devices, there is a procedural challenge caused by the 3 leaflet configuration of the tricuspid valve. The only currently available transcatheter device to perform EET in the tricuspid valve is the MitraClip System, which was initially developed to treat mitral regurgitation and enables grasping of 2 leaflets only. Consequently, more than $1 \mathrm{clip}$ needs to be applied to attach the 3 tricuspid leaflets with each other. In addition, grasping of the leaflets in the center of the tricuspid valve might be difficult in the presence of huge annular dilation and thus a wide gap between the 
leaflets. Nevertheless, considering previous reports and this ex vivo study, central EET is a promising option to mitigate severe TR, especially in complex tricuspid lesions.

In contrast to the clover technique, paracommissural EET has not been used and evaluated in surgical approaches, but was applied in the reported cases of transcatheter tricuspid repair with the MitraClip System. ${ }^{13,15}$ In addition to this clinical aspect and as confirmation of its importance, Vismara and colleagues ${ }^{3}$ evaluated the paracommissural EET, among other techniques, in an ex vivo model. The comparison of this author group between different clip placements and numbers revealed no improvement in valve continence for paracommissural EET only. But in contrast to the present ex vivo investigation, Vismara and colleagues ${ }^{3}$ applied the paracommissural EET to 1 or 2 commissures only and did not evaluate clipping of all 3 commissures. Of note, clipping of all 3 commissures in the conducted ex vivo study resulted in a significant $17.2 \%$ reduction of $\mathrm{v}_{\mathrm{r}}$ in the tethering group. These results are underlined by Hammerstingl and colleagues, ${ }^{13}$ who performed transcatheter tricuspid repair by performing EET in between all 3 commissures in a small case series, leading to improved clinical conditions and acute reduction of TR in all patients. Consequently, paracommissural EET in between all 3 tricuspid commissures might be a promising transcatheter approach to mitigate TR in specific valve pathologies.

In line with this, a recently published clinical study of Nickenig and colleagues, ${ }^{19}$ who investigated transcatheter tricuspid repair with the MitraClip system in 64 patients with FTR, degenerative or mixed TR, showed promising results. These authors reported a significant reduction of $\mathrm{v}_{\mathrm{r}}$ and an improved New York Heart Association Class. Compared with the results of the conducted ex vivo study, the reduction of $\mathrm{v}_{\mathrm{r}}$ was approximately $50 \%$ and thus significantly higher, potentially implicating an underestimation of $v_{r}$ reduction in the ex vivo model compared with clinical conditions. This might be due to the limitations of this ex vivo model, especially the use of physiologic saline instead of blood and sutures for EET instead of clips. However, it has to be highlighted that Nickenig and colleagues ${ }^{19}$ did not investigate postprocedural $\delta \mathrm{p}$, which might be increased because of a reduced orifice area.

\section{Increase of the Mean Transvalvular Gradient}

In all 4 groups, EET resulted in a significant increase in $\delta \mathrm{p}$ without a significant difference between central and paracommissural EET. Considering $\delta \mathrm{p}$ after transcatheter tricuspid repair, only few data are available. Author groups evaluating clinical data ${ }^{14,18}$ and ex vivo data ${ }^{3}$ indicate no more than slight increases of $\delta \mathrm{p}$ without further significance.

In the conducted ex vivo model, central and paracommissural EET resulted in a decrease of the tricuspid valve area, which consequently leads to an increase in $\delta$ p. Considering central EET, these findings are confirmed by clinical studies evaluating the surgical clover technique, ${ }^{12,24,25}$ reporting slight postprocedural increases of $\delta \mathrm{p}$. However, the highest increase in $\delta \mathrm{p}$ was seen after paracommissural EET in the tethering group with a value of $4.4 \pm 3.3 \mathrm{~mm} \mathrm{Hg}$.

All in all, the measured values for $\delta \mathrm{p}$ in the conducted study are slightly higher compared with the previous reports, which is likely caused by the simulation of PAH. Nevertheless, the mean values for $\delta \mathrm{p}$ are not in the range of hemodynamic relevance as defined by the current guidelines. $^{7,8}$

\section{Study Limitations}

The limitations of this study result from using an ex vivo model, which can only provide an approximation to physiologic conditions. Thus, physiologic saline was used instead of blood. The lower viscosity of physiologic saline potentially leads to an increased $v_{r}$. The specific model of this ex vivo study caused TR only by dislocation of the papillary muscles without simulating tricuspid annular dilation. Because concomitant annular dilation is almost always found in TR, this might influence the results of this study. Furthermore, EET was simulated by using surgical suturing techniques, whereas transcatheter techniques in clinical application currently use clipping devices. This is likely to cause a divergence in the reduction of $\mathrm{v}_{\mathrm{r}}$ because clipping devices such as the MitraClip grasp more leaflet tissue, potentially leading to a higher reduction of $\mathrm{v}_{\mathrm{r}}$ compared with sutures. Therefore, this ex vivo model allows for a comparison of different EET among themselves but not for transferring the results directly to clinical practice of clipping.

\section{CONCLUSIONS}

This ex vivo study, with its specific experiment design, provides a significant reduction of TR after EET. It remains uncertain whether the extent of this reduction is sufficient to treat severe TR successfully in clinical practice. In addition, the reduction of TR was achieved at the cost of a significant increase in transvalvular gradients; however, its clinical relevance seems low. Transcatheter approaches need to be evaluated further, especially with regard to concomitant annuloplasty, to improve interventional success.

\section{Webcast}

You can watch a Webcast of this Mitral Conclave meeting presentation by going to: https://aats.blob.core.windows. net/media/17MC/2-MurryE-0725-stock-v1.mp4.

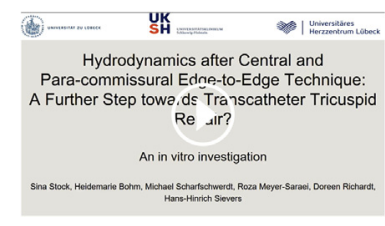




\section{Conflict of Interest Statement}

Authors have nothing to disclose with regard to commercial support.

The authors thank Michael Diwoky for the excellent data management and analyses and for assistance in preparing this article for publication. The authors also thank Richard Börner for support in data analyses.

\section{References}

1. Rogers JH, Bolling SF. The tricuspid valve: current perspective and evolving management of tricuspid regurgitation. Circulation. 2009;119:2718-25.

2. Dreyfus GD, Martin RP, Chan KMJ, Dulguerov F, Alexandrescu C. Functional tricuspid regurgitation. J Am Coll Cardiol. 2015;65:2331-6.

3. Vismara R, Gelpi G, Prabhu S, Romitelli P, Troxler LG, Mangini A, et al. Transcatheter edge-to-edge treatment of functional tricuspid regurgitation in an ex vivo pulsatile heart model. J Am Coll Cardiol. 2016;68:1024-33.

4. Taramasso M, Pozzoli A, Guidotti A, Nietlispach F, Inderbitzin DT, Benussi S, et al. Percutaneous tricuspid valve therapies: the new frontier. Eur Heart J. 2017;38:639-47.

5. Bouleti C, Juliard J-M, Himbert D, Iung B, Brochet E, Urena M, et al. Tricuspid valve and percutaneous approach: no longer the forgotten valve! Arch Cardiovasc Dis. 2016;109:55-66.

6. Nath J, Foster E, Heidenreich PA. Impact of tricuspid regurgitation on long-term survival. J Am Coll Cardiol. 2004:43:405-9.

7. Nishimura RA, Otto CM, Bonow RO, Carabello BA, Erwin JP, O'Gara PT, et al. 2014 AHA/ACC guideline for the management of patients with valvular heart disease: a report of the American College of Cardiology/American Heart Association Task Force on Practice Guidelines. J Thorac Cardiovasc Surg. 2014;148: e1-132.

8. Joint Task Force on the Management of Valvular Heart Disease of the European Society of Cardiology (ESC), European Association for Cardio-Thoracic Surgery (EACTS), Vahanian A, Alfieri O, Andreotti F, Antunes MJ, BarónEsquivias G, Baumgartner H, et al. Guidelines on the management of valvular heart disease (version 2012). Eur Heart J. 2012;33:2451-96.

9. Kim JB, Jung S-H, Choo SJ, Chung CH, Lee JW. Clinical and echocardiographic outcomes after surgery for severe isolated tricuspid regurgitation. J Thorac Cardiovasc Surg. 2013;146:278-84.

10. Bernal JM, Morales D, Revuelta C, Llorca J, Gutiérrez-Morlote J, Revuelta JM. Reoperations after tricuspid valve repair. J Thorac Cardiovasc Surg. 2005;130: 498-503.

11. Kim Y-J, Kwon D-A, Kim H-K, Park J-S, Hahn S, Kim K-H, et al. Determinants of surgical outcome in patients with isolated tricuspid regurgitation. Circulation. 2009; 120:1672-8

12. Lapenna E, De Bonis M, Verzini A, La Canna G, Ferrara D, Calabrese MC, et al. The clover technique for the treatment of complex tricuspid valve insufficiency: midterm clinical and echocardiographic results in 66 patients. Eur J Cardio thorac Surg. 2010;37:1297-303.

13. Hammerstingl C, Schueler R, Malasa M, Werner N, Nickenig G. Transcatheter treatment of severe tricuspid regurgitation with the MitraClip system. Eur Heart J. 2016;37:849-53.

14. Hausleiter J, Braun D, Orban M, Orban M, Mehilli J, Englmaier A, et al. TCT-87 Transcatheter treatment of severe tricuspid regurgitation using the MitraClip ${ }^{\circledR}$ system: 30-day clinical results in 13 consecutive patients. J Am Coll Cardiol. 2016;68:B35-6.

15. Wengenmayer T, Zehender M, Bothe W, Bode C, Grundmann S. First transfemoral percutaneous edge-to-edge repair of the tricuspid valve using the MitraClip system. EuroIntervention. 2016;11:1541-4.

16. Schueler R, Malasa M, Hammerstingl C, Nickenig G. Transcatheter interventions for tricuspid regurgitation: MitraClip. EuroIntervention. 2016;12:Y108-9.

17. Lesevic H, Frangieh AH, Kasel AM, Ott I. Successful percutaneous edge-to-edge repair in degenerative tricuspid valve regurgitation using the MitraClip system. Eur Heart J. 2017:38:691.

18. Kowalski M, Franz N, Hofmann S, Ritter F, Thale J, Warnecke H. Transapical transcatheter aortic valve implantation followed by transfemoral transcatheter edge-to-edge repair of the tricuspid valve using the MitraClip system - a new treatment concept for an inoperable patient with significant aortic stenosis and severe tricuspid valve regurgitation. Postepy Kardiol Interwencyjnej. 2016;12: 262-6.

19. Nickenig G, Kowalski M, Hausleiter J, Braun D, Schofer J, Yzeiraj E, et al Transcatheter treatment of severe tricuspid regurgitation with the edge-to-edge MitraClip technique. Circulation. 2017;135:1802-14.

20. Lancellotti P, Moura L, Pierard LA, Agricola E, Popescu BA, Tribouilloy C, et al European Association of Echocardiography recommendations for the assessment of valvular regurgitation. Part 2: mitral and tricuspid regurgitation (native valve disease). Eur J Echocardiogr. 2010;11:307-32.

21. Scharfschwerdt M, Misfeld M, Sievers H-H. The influence of a nonlinear resis tance element upon in vitro aortic pressure tracings and aortic valve motions Asaio J. 2004;50:498-502.

22. Campelo-Parada F, Perlman G, Philippon F, Ye J, Thompson C, Bédard E, et al First-in-man experience of a novel transcatheter repair system for treating severe tricuspid regurgitation. J Am Coll Cardiol. 2015;66:2475-83.

23. Alfieri O, De Bonis M, Lapenna E, Agricola E, Quarti A, Maisano F. The "clove technique" as a novel approach for correction of post-traumatic tricuspid regur gitation. J Thorac Cardiovasc Surg. 2003;126:75-9.

24. De Bonis M, Lapenna E, La Canna G, Grimaldi A, Maisano F, Torracca L, et al. A novel technique for correction of severe tricuspid valve regurgitation due to complex lesions. Eur J Cardio-Thorac Surg. 2004;25:760-5.

25. Lai Y-Q, Meng X, Bai T, Zhang C, Luo Y, Zhang Z-G. Edge-to-edge tricuspid valve repair: an adjuvant technique for residual tricuspid regurgitation. Ann Thorac Surg. 2006;81:2179-82.

Key Words: tricuspid regurgitation, transcatheter tricuspid repair, ex vivo 